\title{
GALAXY FORMATION: ISOCAM COUNTS
}

\author{
C.J. CESARSKY AND D. ELBAZ \\ Service d'Astrophysique \\ CEA, DSM, DAPNIA, CE Saclay, F91191 Gif-Sur-Yvette cedex, \\ France
}

\section{Introduction}

Detailed observations in the optical do not allow to discriminate between the following scenarii of galaxy formation:

- synchronous formation at large redshift $(z>7)$.

- hierarchical merging, down to $z \leq 1$, of proto-galactic lumps formed at very high redshift $(\approx 10)$.

- galaxy formation at low $\mathrm{z}(\approx 2-5)$, but partially obscured by dust.

More than 1500 galaxies in a field of $2.6^{\prime} \times 4.6^{\prime}$ were detected by Tyson (1988) with CCD images in the B band. These deep counts of galaxies revealed the presence of an unexpectedly large population of blue galaxies, corresponding to a number excess of 3 to 5 times more galaxies at $\mathrm{B}>23$ than one can expect from the local luminosity function. These objects lie apparently at moderate redshift $(\mathrm{z}=0.3$ for $\mathrm{B}=23-24)$ as shown by spectroscopy of B-selected galaxies (Colless et al. 1990).

Multiplexing spectroscopy with the MOS (multi-object spectrograph) at CFHT confirmed this result, but also showed that up to $50 \%$ of the faint blue galaxies $(\mathrm{B}=24)$ are associated to narrow emission line AGNs (Tresse et al. 1994) rather than to starburst galaxies as previously stated.

In their discussion about the origin of this population of blue galaxies at moderately low redshift, Colless et al. (1993) reviewed different models of galaxy formation and evolution, and concluded that the current observations do not allow to discriminate between these models, although no-evolution models seemed to be best suited.

A separate issue is that of the intrinsically bright and distant blue galaxies, which were found to be absent in Colless et al. (1993) redshift surveys made using the Low Dispersion Survey Spectrograph (LDSS) on the Anglo- 
Australian Telescope (AAT), with CCD photometry in B, R and I bands. Tresse et al. (1994), Hammer et al. (1994) found that these galaxies were brighter by several tens of magnitude when $z>0.5$, hence showing evidence of evolution.

As pointed out several years ago by Ostriker and Heisler (1984), distant objects may be significantly obscured by dust at optical wavelengths, either by surrounding dust, dust in intervening galaxies, or some combinaison of these. Thus (see also Colless et al. 1993), infrared-selected redshift surveys are mandatory to go one step further towards the understanding of galaxy formation and evolution. Two teams intend to carry out deep surveys within the Central programme of the ISO satellite: Cesarsky et al. and Taniguchi et al. (see references).

\section{Infrared Surveys}

Even if they go much less deep than Tyson's (1988) survey, surveys in the thermal infrared are decisive in the determination of the process of galaxy fomation. Indeed, as pointed out by De Zotti et al. (1994), three effects concur in easing the detection of high-z galaxies at mid-IR wavelengths:

- the decreasing effect of dust absorption as compared to shorter wavelengths.

- the positive $\mathrm{K}$-correction implied by galaxy spectra raising up to $\approx 1$ $\mu \mathrm{m}$.

- the positive luminosity evolution.

In this context, a mid-IR survey will allow to test:

- the presence of "slightly dusty" proto-galaxies, i.e. galaxies where most of the MIR emission comes from the redshifted stellar emission rather than from dust radiation.

- the luminosity evolution of spiral galaxies (either starbursts or not).

- the possibility that ellipticals are formed in a strong burst of star formation at $\mathrm{z}=2$ to 5 (in a phase similar to that observed for IRAS $\mathrm{F} 10214+4724$, at $\mathrm{z}=2.3$, Rowan-Robinson et al. 1991).

- the density of low-luminosity AGNs $(\approx 15 \mu \mathrm{m})$.

- the origin of quasars. Are quasars born in strong galaxy interactions, in conjonction with an active starburst phase (Sanders 1988, Lipari et al. 1994)?

\section{The Infrared Space Observatory, ISOCAM and ISOPHOT}

In 1983, just as the first results of IRAS were presented to the european astronomical community, the decision was taken at the European Space Agency to fly a second generation infrared cryogenic satellite, ISO (the Infrared Space Observatory). While IRAS was scanning the whole sky in 


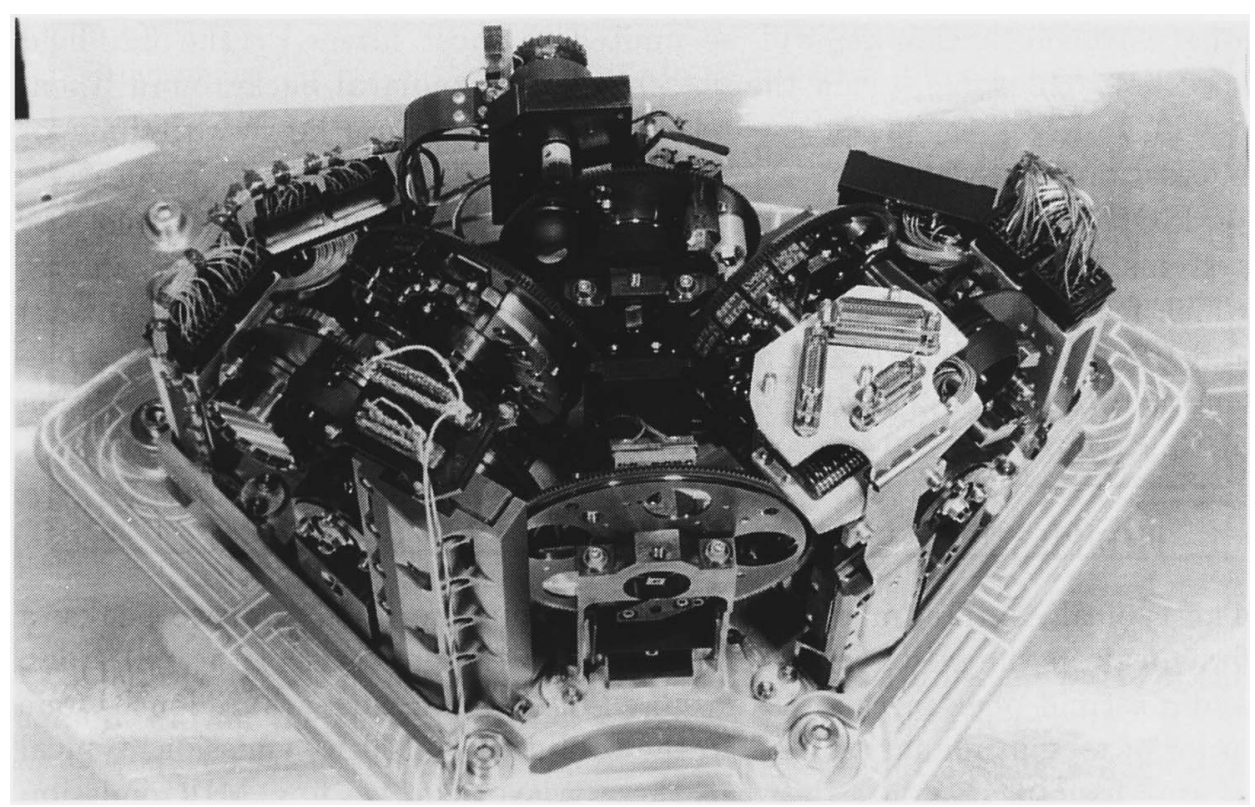

Figure 1. ISOCAM IR camera

four colours, ISO is destined to perform detailed studies of selected regions, with better angular resolution, wider wavelength coverage, enhanced imaging and spectroscopic capabilities, and a higher sensitivity. ISO will be launched in September 1995.

Four instruments have been placed in the focal plane of the ISO telescope: a camera (ISOCAM), a photometer (ISOPHOT), and two spectrometers (SWS and LWS), which, together, cover the range 2.5 to $200 \mu \mathrm{m}$. Two of them will be used in the deep surveys of the ISO Central Programme: ISOCAM and ISOPHOT.

ISOCAM (Cesarsky et al. 1994) is a two way camera, featuring two $(32 \times 32)$ array detectors, one for short wavelengths ( $\mathrm{SW}, 2.5$ to $5.5 \mu \mathrm{m})$, the other for long wavelengths (LW, 4 to $17 \mu \mathrm{m}$ ). On each channel, there are two wheels, one carrying four lenses, allowing four different pixels fiels of view (1.5", 3", 6", 12"), and the other carrying 10 to 12 fixed filters and one or two Circular Variable Filters (CVF), allowing to reach a spectral resolution of about 45. A wheel at the entrance has four positions: 3 polarizers and a hole. A selection wheel carries Fabry mirrors which can direct the light beam of the ISO telescope towards one or the other of the detectors, or illuminate them uniformly with an internal calibration source, for flat field purposes. The picture of ISOCAM is shown on figure 1 .

With exposures of a few minutes, the long wavelength detector will 
easily detect sources at the sub-mJy level, and the short wavelength channel sources at the mJy range. With longer exposures, the sensitivity of the long wavelength channel will be limited in most filters by the flat field accuracy achievable, given the presence of the zodiacal background; then, it will be necessary, as in ground observations, to use beam switching or microscanning techniques.

ISOPHOT (Lemke et al. 1994) is a complex instrument, with three subsystems. Only one of them will be used in the Deep Survey: the camera, which features two arrays made of individual detectors: C100 $(3 \times 3$ pixels $)$ and C200 ( $2 \times 2$ pixels), and allows to take images of the sky in the wavelength range 50 to $240 \mu \mathrm{m}$, with various filters and an angular resolution of $83.9 "\left(d_{\text {Airy }}\right)$ at $100 \mu \mathrm{m}$ and $168 "$ at $200 \mu \mathrm{m}$.

\subsection{THE PHYSICS}

The typical continuum emission of galaxies in the IR shows two peaks: a first peak in the NIR, around $1 \mu \mathrm{m}$, due to the emission of the red stars, and a second peak in the FIR, around $100 \mu \mathrm{m}$ and with a flux density one order of magnitude larger, due to the cool dust $(<100 \mathrm{~K})$ emission, typical of star forming regions. Between these two peaks, we find MIR emission from hot dust, around $10 \mu \mathrm{m}$, and NIR-MIR emission, of PAHs.

Given the relatively coarse resolution that can be obtained, even at diffraction limit in the infrared with a $60 \mathrm{~cm}$ telescope, and the great sensitivity obtained with a cryogenic system, the confusion limit may be a concern even with ISOCAM. With a 6 " pixel field of view, the $5 \sigma$ confusion limit for the galaxies in the two most sensitive ISOCAM filters is respectively 20 and $30 \mu \mathrm{Jy}$ for the 6.7 and $12 \mu \mathrm{m}$ bands, using the count prediction by Franceschini et al. (1991).

\subsection{THE OBSERVATION PROGAMMES}

Two galaxy count programmes have been scheduled in the ISO Central Programme using ISOCAM and ISOPHOT (see table 1):

- Cesarsky et al. (see references) Deep Survey programme (CCESARSK. IDSPCO) consists in long and repeated integrations in broad and sensitive CAM (LW2: 5-8 $\mu \mathrm{m}$, LW3: 12-18 $\mu \mathrm{m}$ ) and PHOT (C100: $90 \mu \mathrm{m}$ ) filters. The survey will be performed at two different depths with CAM, with a pixel field of view of 6 ": a "deep" survey (DS) with both filters, with a $4 \sigma$ sensitivity of $34 \mu \mathrm{Jy}$ for LW2 and $13 \mu \mathrm{Jy}$ for LW3, and a "shallow" survey (SS) with only LW3, for a lower sensitivity of $38 \mu \mathrm{Jy}$. Two fields will be mapped, the "Marano" field (MF, southern field) and the "Lockman" hole (LH, northern field), with a large area for the SS (about 1 square degree) and a smaller area for the DS (0.17 and 0.11 sq. deg. for MF and 


\section{TABLE 1.}

\begin{tabular}{|c|c|c|c|c|}
\hline Programme & \multicolumn{3}{|c|}{$\begin{array}{l}\text { CCESARSK. } \\
\text { IDSPCO }\end{array}$} & $\begin{array}{l}\text { YTA NIGUC. } \\
\text { DEEPPGPO }\end{array}$ \\
\hline & SS-L.W3 & DS-LW3 & DS-I.W2 & LW2 \\
\hline $\begin{array}{l}4 \sigma \text { sensitivity } \\
\text { (point source) } \\
\mu \mathrm{Jy}\end{array}$ & 380 & 130 & 34 & 10 \\
\hline Total time (h) & $\begin{array}{l}\text { MF: } 20 \\
\text { LH: } 14\end{array}$ & $\begin{array}{l}\text { MF: } 20 \\
\text { LH: } 13\end{array}$ & $\begin{array}{l}\text { MF: } 30 \\
\text { LH: } 20\end{array}$ & LH: 30 \\
\hline $\begin{array}{l}\text { Number of } \\
\text { CAM beams } \\
\left(3^{1} \times 3^{\prime}\right)\end{array}$ & $\begin{array}{l}\text { MF: } 350 \\
\text { LH: } 250\end{array}$ & $\begin{array}{l}\text { MF: } 60 \\
\text { LH: } 40\end{array}$ & $\begin{array}{l}\text { MF: } 60 \\
\text { LH: } 40\end{array}$ & LH: 4 \\
\hline $\begin{array}{l}\text { Area covered } \\
\text { (sq. degree) }\end{array}$ & $\begin{array}{l}\text { MF: } 1.0 \\
\text { LH: } 0.7 \\
\end{array}$ & $\begin{array}{l}\text { MF: } 0.17 \\
\text { LH: } 0.11 \\
\end{array}$ & $\begin{array}{l}\text { MF: } 0.17 \\
\text { LH: } 0.11 \\
\end{array}$ & LH: 0.01 \\
\hline
\end{tabular}

LH respectively). The same pixel of the sky is seen by different parts of the camera during the survey in order to detect any systematic effects.

A long wavelength map of the Southern SS (about 1 square degree in the Marano field, for a total observation time of 20 hours) with ISOPHOT C100 at 90 microns will be achieved in the same observing mode than the one described afterwards for the Taniguchi et al. proposal.

- Taniguchi et al. (see references) Search for Primeval Galaxies and Quasars programme (YTANIGUC.DEEPPGPQ). The survey will be performed at one single depth, with a pixel field of view of 3" and the LW2 filter, hoping to reach a $4 \sigma$ sensistivity of $10 \mu \mathrm{Jy}$. The area covered will be small: 0.01 square degree. A 90 and $160 \mu \mathrm{m}$ map will be performed with the oversampling mode (PHT32) of PHOT in about 2.4 square degree in the Lockman Hole. The sensitivity at a 5 sigma level is expected to be $6.7 \mathrm{mJy}$ for the $90 \mu \mathrm{m}$ filter and $13 \mathrm{mJy}$ for the $160 \mu \mathrm{m}$ filter (for a total observation time of 28 hours). It is slightly below the confusion limit due to galaxies ( $7 \mathrm{mJy}$ and $10 \mathrm{mJy}$ for respectively 90 and $160 \mu \mathrm{m})$ and well above the confusion due to cirrus clouds (because the region is selected in a dark IRAS area). This survey will cover most or all of the area surveyed by Cesarsky et al. at shorter wavelengths.

\subsection{PREDICTIONS FOR ISOCAM COUNTS}

In order to estimate the observational feasibility of this survey, Taniguchi et al. estimated their galaxy fluxes using the Model B of Larson (1974) where a protogalactic gas cloud is assumed to collapse in a free-fall time, resulting in an intense star formation phase in the nuclear region; the in- 
frared emission results mainly from the shifted emission of the stars. They expect to detect about 3 primeval galaxies, in the case of 1 mag extinction $\left(E_{B-V}=1\right)$. About 30 primeval quasars are expected to be found with the PHOT survey.

On the other hand, Cesarsky et al. used the different model of Franceschini et al. (1991, De Zotti et al. 1994) to make predictions on the galaxy counts. This model takes into account the reprocessing of the stellar emission by dust that re-radiates it in the IR. With this approach, they expect to find about 400 galaxies in the whole survey with the LW2 filter (DS only), where the contribution of elliptical galaxies is not dominant. Two possibilities have been tested in this model, for the survey in the LW3 filter, depending on the assumption that ellipticals show evolution or not: the total galaxy counts in the DS (and SS) is equal to 315 (respectively 486), if evolution is assumed, and 81 (respectively 189), if no evolution is assumed. Maffei et al. (1994) have considered the influence of a more detailed modelisation of the PAHs emission, including emission lines, and found a slightly different estimation of the expected galaxy counts (with no galaxy evolution). Due to the K correction, the PAHs emission is shifted towards higher wavelength, increasing the galaxy counts in the LW2 and LW3 filters. When comparing the predictions of Franceschini et al. and Maffei et al.:

- for LW2, the two $\log N-\log S$ curves cross each other around the sensitivity of $S=0.1 \mu \mathrm{Jy}$, but Maffei's model predicts one order of magnitude more counts for a sensitivity of $\mathrm{S}=0.1 \mathrm{mJy}$.

- for LW3, Maffei's model predicts about 3 times more galaxies than Franceschini (1991), whatever the sensitivity.

\subsection{WILL THESE SURVEYS REVEAL MORE FAR-AWAY, ULTRALUMINOUS SOURCES?}

With the IRAS survey and its follow up at various wavelengths, the importance of the role of galaxy interactions in the formation of ultraluminous galaxies galaxies was established (Sanders et al. 1988). Moreover, if these objects were more common in the past (Sanders et al. 1988; RowanRobinson et al. 1991), we would expect to detect at least several of them at high $z$ with the more sensitive ISO surveys.

Mrk 231 is the nearest object $(z=0.0269)$ of this kind showing extreme IR emission and where the bolometric luminosity is dominated by the IR continuum emission (Lipari et al. 1994). Such a galaxy could be detected as far as $z=3$ with the DS (CAM LW2 and LW3), $z=2$ with the SS (for CAM LW3), and $z=1$, for PHOT at $90 \mu \mathrm{m}$.

Similarly, figure 3 shows the depth at which a typical elliptical galaxy with $M_{B} \approx 20$ could be detected with ISO: the LW2 DS goes deeper than $\mathrm{z}=1$, while for LW2 the DS gets close to $\mathrm{z}=0.5$ and the SS to $\mathrm{z}=0.2$. 


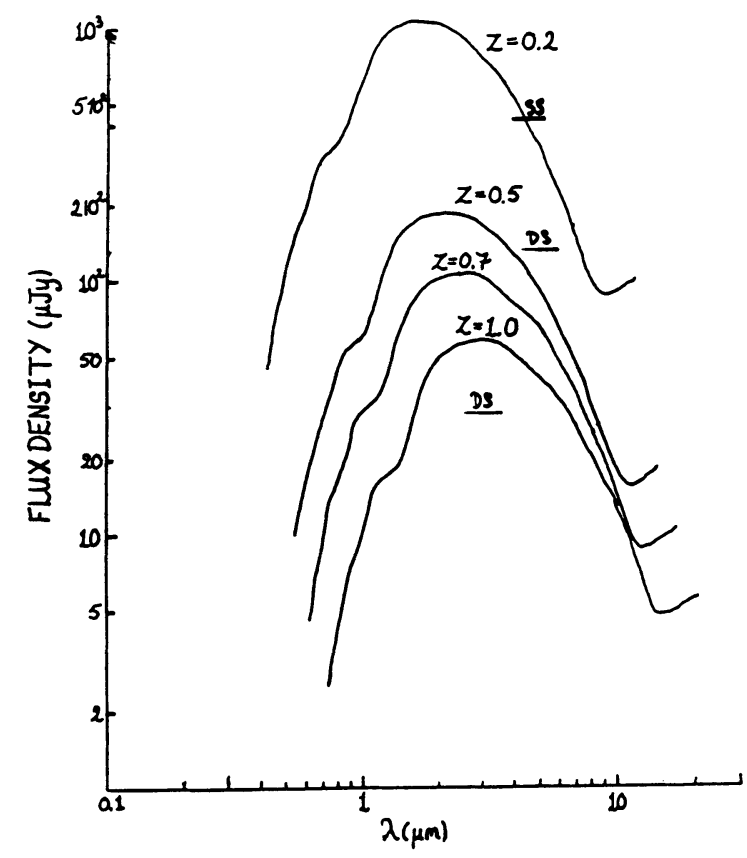

Figure 2. Spectrum of a typical E/SO galaxy with $M_{B} \approx 20$ transposed to various redshifts with no significant evolution (from S. Chase)

A completely different approach seems to favor the hypothesis that these surveys can also detect very young spheroidal galaxies. Indeed it is now well-known that the intergalactic gas in clusters of galaxies (intracluster medium, ICM) has been enriched by the galaxies with an iron mass at least equal to that contained in the galaxies (Renzini et al. 1993). This Fe mass being directly proportional to the total luminosity of ellipticals (E/SO) only, it was suggested to originate from these galaxies (Arnaud et al. 1992). To process such a large amount of iron and eject it into the ICM, E/SO must have experienced a strong starburst at the scale of the whole galaxy, with an IMF shifted towards massive stars (Elbaz et al. 1994). The SNII origin of the intergalactic iron has recently been confirmed by the detection in X-ray of an overabundance of O over Fe, typical of SNII (Mushotzky 1994, to appear) and a low abundance (solar or less) of the hot corona of three bright ellipticals by ROSAT (Forman et al. 1993) and ASCA (Awaki et al. 1994), much too low for an SNIa origin of the iron. Such a model has been applied to the hyperluminous galaxy 10214+4724 (Elbaz et al. 1992) and predicts an intense and very brief phase (a few hundred million years only) of high-mass stars formation, that should be detectable in the IR at relatively high redshift. 


\section{References}

Arnaud, M., Rothenflug, R., Boulade, O., Vigroux, L., Vangioni-Flam, E., 1992, A\&A 254, 49

Awaki, H., et al., 1994, PASJ 46, L65

Cesarsky, C.J., 1993, Proceedings of the XXVIIIth Rencontre de Moriond: "The Cold Universe", eds. Montmerle, Th., Lada, Ch.J., Mirabel, I.F., Trân Thanh Vân, Ed. Frontieres, p.417

Cesarsky, C.J., Chase, S., Danese, L., Desert, F.X., Franceschini, A., Harwitt, M.O., Hauser, M., Koo, D., Mandolesi, N., Puget, J.-L., ISO Central Progamme: CCESARSK.IDSPCO

Cesarsky, C.J., et al., 1994, Opt. Eng. 33, 751

Colless, M., Ellis, R., Taylor, K., Hook, R., 1990, MNRAS 235, 827

Colless, M., Ellis, R.S., Broadhurst, T.J., Taylor, K., Peterson, B.A., 1993, MNRAS 261, 19

De Zotti, G., Franceschini, A., Mazzei, P., Toffolatti, L., Danese, L., 1994, to be published.

Elbaz, D., Arnaud, M., Casse, M., Mirabel, I.F., Prantzos, N., VangioniFlam, E., 1992, A\&A 265, L29

Elbaz, D., Arnaud, M., Vangioni-Flam, E., 1994, to appear in A\&A

Forman, W., Jones, C., David, L., Franx, M., Makishima, K., ohashi, T., 1993, ApJ 418, L55

Franceschini, A., Toffolatti, L., Mazzei, P., Danese, L., De Zotti, G., 1991, A\&AS 89, 285

Hammer, F., Lilly, S., Le Fevre, O., Crampton, D., Tresse, L., 1994, in Proceedings of the ESO workshop: Science with the VLT, Munchen 28th June, Eds. Walsh \& Danziger, Springer-Verlag, in press.

Larson, R.B.,1974, MNRAS 166, 585

Lemke, D., et al., 1994, Opt. Eng. 33, 20

Lipari, S., Colina, L, Machetto, F., 1994, to appear in ApJ

Maffei, B., Puget, J.-L., Desert, F.X., 1994, in preparation.

Ostriker, J.P., Heisler, J., 1984, ApJ 278,1

Renzini, A., Ciotti, L., D'Ercole, A., Pellegrini, S., 1993, ApJ 419, 52

Rowan-Robinson, M., et al., 1991, Nature 351, 719

Sanders, D.B., Soifer, B.T., Elias, J.H., Matthews, K., Madore, B.F., 1988, ApJ 325, 74

Taniguchi, Y., Okuda, H., Matsumoto, T., Wakamatsu, K., Kawara, K., Cowie, L., Joseph, R., Sanders, D., Chambers, K., Wynn-Williams G., Sofue, Y., Matsuhara, H., Sato, Y., Desert, X., ISO Central Progamme: YTANIGUC.DEEPPGPQ

Tresse, L., Rola, C., Hammer, F., Stasinska, G., 1994, in preparation.

Tyson, J., 1988, AJ 96, 1 